\title{
ATOMIC IODINE LASER UTILIZED HIGH-PRESSURE PULSED SINGLET OXYGEN GENERATOR
}

\author{
M. ENDO, K. SHIROKI and T. UCHIYAMA \\ Department of Electrical Engineering, Faculty of Science and Technology, Keio University, \\ 3-14-1 Hiyoshi, Kohoku-ka, Yokohama 223, Japan
}

\begin{abstract}
A pulsed iodine laser driven by a chemical oxygen generator was developed. The energy deposition on $\left.I_{\left({ }^{2}\right.} \mathrm{P}_{3} / 2\right)$ was made by a high pressure pulsed $\mathrm{O}_{2}\left(\mathrm{a}^{1} \Delta \mathrm{g}\right)$ generator. Although this scheme is same as the conventional Chemically pumped Oxygen-Iodine Lasers (COIL), it is unique in that the pulsed laser was extracted by the high-energy density pulsed pumping scheme. The pulse energy obtained was $2.46 \mathrm{~J}$, the maximum peak power obtained was $900 \mathrm{w}$. Pulse width was ranging from $2 \mathrm{~ms}$ to $5 \mathrm{~ms}$. The specific energy was estimated to be $7.7 \mathrm{~J} / 1$.
\end{abstract}

\section{1:Introduction}

The iodine photodissociation laser is one of the most high-power pulsed lasers. The largest system emits $1.2 \mathrm{~kJ}$ output with a sub-nanosecond pulse width[1], and it is utilized for studies of laser nuclear fusion, X-lay lasers, and other plasma physics[2][3] such as glass lasers and eximer lasers. The advantage of gas lasers against glass lasers is that operation cycle can be much faster because they are free from the thermal lensing problem. There are iodine lasers in which an iodine atom is pumped by energy transfer from chemically generated $\mathrm{O}_{2}\left(\mathrm{a}^{1} \Delta \mathrm{g}\right)$. These are known as COIL(Chemically pumped oxygen-Iodine Laser). COIL operates in $\mathrm{CW}$ regime and several tens of kilowatts of output power is available[4]. There have been a proposal to utilize this laser for an amplifier of the iodine laser chain[5], but realistic systems have not been reported yet. The main problem is that most $\mathrm{O}_{2}\left(\mathrm{a}^{1} \Delta_{\mathrm{g}}\right)$ generators can only work under fairly low pressures (lower than a few Torr) and the iodine pressure isn't allowed to be higher than one hundredth of the oxygen pressure by the oxygen-iodine kinetics. Thus, most of the medium energy is stored in the oxygen molecules and gradually transferred to the iodine atoms. As the characteristic time of the energy transfer is inversely proportional to the iodine pressure, higher iodine pressure is required for pulsed energy extraction. To avoid this limitation, Basov, et. al. reported[6] that if an iodine containing substance such as $\mathrm{CH} 3 \mathrm{I}$ is mixed with $\mathrm{O}_{2}\left(\mathrm{a}^{1} \Delta_{\mathrm{g}}\right)$ and dissociated by the flashlamp radiation, the $\mathrm{I} / \mathrm{O}_{2}\left(\mathrm{a}^{1} \Delta \mathrm{g}\right)$ limitation will be improved tenfold. The other way to avoid this limitation is to increase the oxygen pressure, which promises an 
increased iodine pressure.

Recently, we have developed a high pressure pulsed Singlet Oxygen Generator(SOG) which produces $\mathrm{O}_{2}\left(\mathrm{a}^{1} \Delta_{\mathrm{g}}\right)$ pressure 10 times higher than conventional soGs[7][8]. The special feature of this device is to utilize a ceramic porous pipe as the reaction zone, which was reported previously[9]. We have been studying an amplifier of a high-power pulsed iodine laser as one of the applications of this device. First of all, we demonstrated the operation of this device as an oscillator.

\section{2: Experiment}

Fig.1 shows the schematic drawing of the apparatus. It consists of SOG, iodine injectors, cavity arms with laser mirrors, and measurement devices. The SOG consists of a solution tank which contains basic hydrogen peroxide, a chlorine tank, and a porous pipe on whose surface $\mathrm{O}_{2}\left(\mathrm{a}^{1} \Delta \mathrm{g}\right)$ generation occurs. The performance of this SOG is that it can fill momentarily the upper volume of SOG $(40 \mathrm{~mm} \times 40 \mathrm{~mm} \times 1000 \mathrm{~mm})$ up to $34 \mathrm{Torr}$ of $\mathrm{O}_{2}\left(\mathrm{a}^{1} \Delta_{\mathrm{g}}\right)$. The properties of this SOG were reported previously[7][8]. There are 35 iodine injector tubes just above the sOG. Each injector is made of a $6 \mathrm{~mm} \phi$ copper tube which has five $0.7 \mathrm{~mm} \phi$ holes on the top. A heated pre-chamber which contains mixture of iodine and argon gas is connected to injectors through a manifold designed to provide the mixture quickly. The laser cavity, $2500 \mathrm{~mm}$ long and $30 \mathrm{~mm} \phi$ in diameter is directly connected to the SOG. The entire system is evacuated and kept under 0.5Torr by a rotary pump with a capacity of 300oliter/min. The operation is conducted as follows; first, V1 and V3 are kept close, Cl2 tank contains about 2200Torr of pure chlorine gas, prechamber contains iodine and argon mixture at a temperature of 400K. Just before the operation, V2 is closed to isolate the cavity from the vacuum system. When V2 was closed, V1 and V3 are opened simultaneously, and $\mathrm{O}_{2}$ and $\mathrm{I}_{2}$ mixture fill the upper volume of the system. As V1 and V3 are pneumatic high-speed butterfly valves, they are opened within 50ms. Although the cavity pressure rises linearly, the $\mathrm{O}_{2}\left(\mathrm{a}^{1} \Delta \mathrm{g}\right)$ pressure saturates, then decays because of strong quenching reactions. Population inversion is formed in a moment and sustained for a few milliseconds. After the operation, V2 is opened and system is evacuated again.

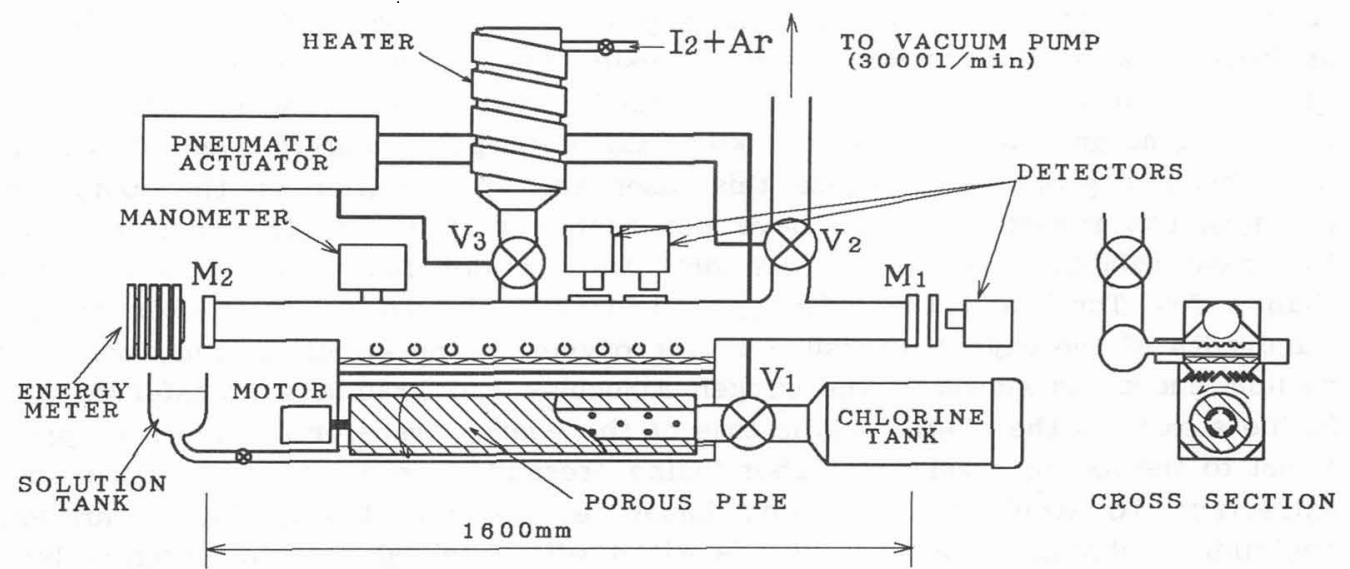

Fig.1 Schematic drawing of the apparatus 
3:Results and discussion

A typical result of an operation is shown in Fig.2. Four bold traces show the laser power, $I^{*}, \mathrm{O}_{2}\left(\mathrm{a}^{1} \Delta \mathrm{g}\right)$, and total pressures. The $I^{*}$ pressure was calculated from the $1315 \mathrm{~nm}$ emission detected by the filtered Ge photodiode, and The $\mathrm{O}_{2}\left(\mathrm{a}^{1} \Delta_{\varepsilon}\right)$ pressure was calculated from the $634 \mathrm{~nm} 2 \mathrm{O}_{2}\left(\mathrm{a}^{1} \Delta \mathrm{g}\right)$ dimole emission detected by the filtered Si photodiode. Stimulated emission started when total pressure was 2030 Torr, and sustained 2-5ms. Because the peak power, pulse width, and output energy of this device was varied by the jitter of valve opening time, iodine temperature, and other factors, the reproducibility of the laser output was bad. The I* emission was strongly interfered by the scattered laser emission. The red emission was intensified when laser emission occurred, but the wavelength and species emitted is unknown. The difference between lased case and not lased case is shown in this figure. The maximum energy obtained was $2.46 \mathrm{~J}$ with $5.2 \mathrm{~ms}$ pulse width, and the maximum peak power obtained was $0.9 \mathrm{~kW}$ with $2.6 \mathrm{~ms}$ pulse width. We took burn patterns just behind the mirror and found that the cross section of the mode is $2.0 \mathrm{~cm}^{2}$. Thus the extracted energy divided by mode volume is calculated to be $7.7 \mathrm{~J} / 1$.

Fig.3 shows the output energy as a function of mirror transmittance. From this graph, the gain of this system is estimated to be $3 \%$ by a round trip. As the cavity length is $1.6 \mathrm{~m}$, the small signal gain is calculated to be $9.4 \times 10^{-5} \mathrm{~cm}^{-1}$.

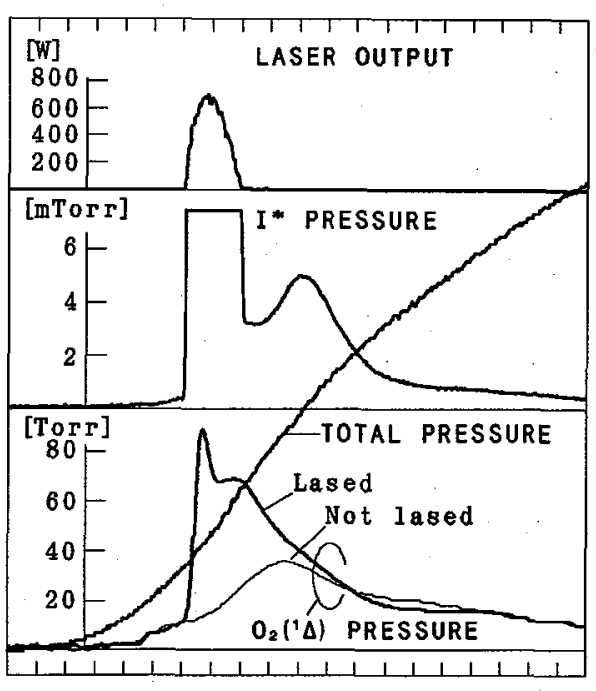

TIME : $2 \mathrm{~ms} / \mathrm{d} i \mathrm{v}$

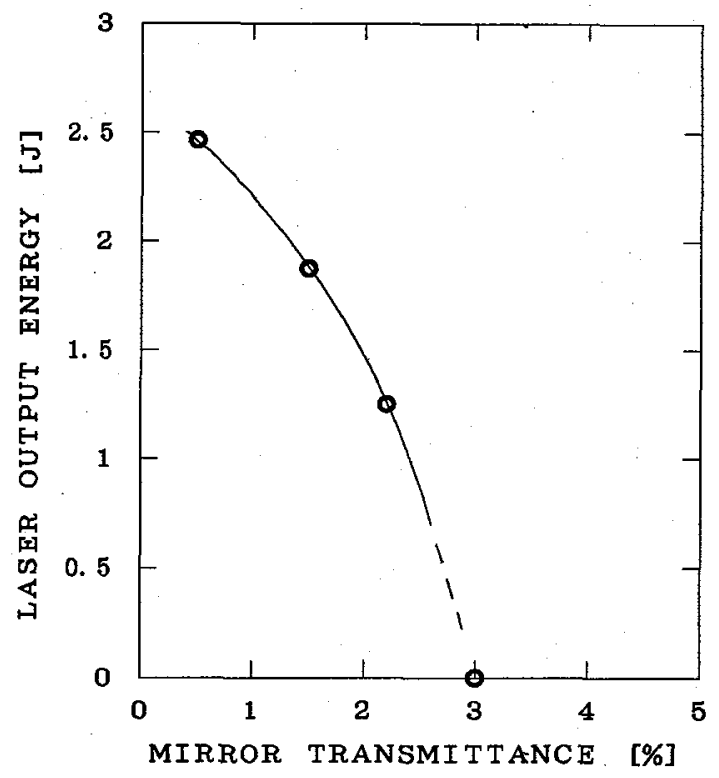

Fig.2 Typical result of an experiment

Fig.3 Laser output as a funtion of mirror transmittacce

The specific energy of $7.7 \mathrm{~J} / 1$ and the small signal gain of $9.4 \times 10^{-5} \mathrm{~cm}^{-1}$ seem to be insufficient to utilize this scheme as a pulse laser amplification. Our theoretical calculations predict that both the extractable energy and small signal gain will be improved by more than 10 times. The most probable reasons for this small values are an insufficient amount of iodine injection, imperfect $\mathrm{O}_{2} / \mathrm{I}_{2}$ mixing, and the time delay of the iodine injection between the nearest and farthest injector 
from the pre-chamber. Because our system was relatively primitive, it didn't incorporate any sophisticated devices for iodine mixing. The second rason why the net gain was low is because there was loss in the cavicy caused by fine water droplets, which are the by-product of the $\mathrm{O}_{2}\left(\mathrm{a}^{1} \Delta_{\mathrm{g}}\right)$ generation.

Incidentally, it should be noted that this system is unique as a coIL system because it doesn't use any water vapor trapping devices. Although we have reported a COIL without water vapor trap previously[10], it required an external solution chiller. In this system, we didn't even employ a solution chiller. In the pulse operation, the energy deposition time is characterized by the time to transfer energy from $\mathrm{O}_{2}\left(\mathrm{a}^{\mathrm{I}} \Delta_{\mathrm{g}}\right)$ to $\mathrm{I}\left({ }^{2} \mathrm{P}_{3 / 2}\right)$ namely,

$$
\tau_{t} \doteqdot 1 /\left(x_{t}[I(2 \mathrm{P} 3 / 2)]\right) \text {. }
$$

Where $\mathbf{K} t=7.6 \times 10^{-11} \mathrm{~cm}^{-3} \mathbf{s}^{-1}$ is the pumping reaction rate constant. In our case, $\tau t$ is estimated to be on the order of $0.1 \mathrm{~ms}$. On the other hand, the characteristic relaxation time of the stored energy is governed by $\mathrm{H}_{2} \mathrm{O}, \mathrm{I}\left({ }^{2} \mathrm{P}_{3} / 2\right)$, and $\mathrm{O}_{2}\left(\mathrm{a}^{1} \Delta_{\mathrm{g}}\right)$ densities as follows;

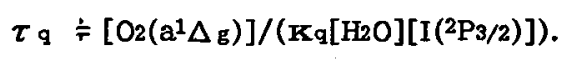

Where $\mathrm{Kq}_{\mathrm{q}}=2 \times 10^{-12} \mathrm{~cm}^{-3} \mathrm{~s}^{-1}$ is the $\mathrm{I}\left({ }^{2} \mathrm{P}_{1 / 2}\right)$ quenching rate constant by $\mathrm{H}_{2} \mathrm{O}[11]$. If no water vapor control is made, $\tau_{\mathrm{q}}$ will be shorter than $1 \mathrm{~ms}$. It is too short for the $\mathrm{CW}$ case, in which medium is transported from the reaction zone to the resonator by the gas flow.

\section{4: S ummary}

In summary, we have demonstrated pulsed iodine laser driven by pure chemical pumping scheme. Output energy and peak power was $2.46 \mathrm{~J}$ and $900 \mathrm{~W}$ respective1y. The specific energy was estimated to be $7.7 \mathrm{~J} / 1$.

We wish to acknowledge productive interaction with Mr. Soichiro Ikeda of Oshitari Laboratory co. Inc.

\section{REFERENCES:}

[1] E. Fill, Max Planck Inst. for Quant. Opt., private communication.

[2] S. Sakabe, R. Sigel, G. D. Tsakiris, I. B. Foldes, and P. Herrmann, Phys. Rev. A 38, 5756 (1988).

[3] R. Sigel, K. Eidmann, F. Lavarenne, and R. F. Schmalz, Phys. Fluids B $\underline{2}_{1} 199$ (1990).

[4] P. V. Avizonis, G. Hasen, and K. A. Truesdell, Proc. SPIE 1225, 448 (1990).

[5] G. N. Hays and G. A. Fisk, IEEE J. Quant. Electron. QE-17, 1823 (1981).

[6] N. G. Basov, P. G. Kryukov, and N. N. Yuryshev, Sov. J. Quant. Electron. 17, 588 (1987).

[7] M. Endo, S. Arai, T. Yamashita, and T. Uchiyama, Proc. SPIE 1225, 439 (1990).

[8] M. Endo, K. Shiroki, and T. Uchiyama, Proc. SPIE 1397, 267 (1990).

[9] K. Takehisa, N. Shimizu, and T. Uchiyama, J. Appl. Phys. 61, 68 (1987).

[10]T. Kikuchi, T. Tsuruyama, and T. Uchiyama, J. Appl. Phys. 64, 2873 (1988).

[11]M. V. Zagidullin, V. I. Igoshin, V. A. Katulin, N. L. Kupriyanov, and

N. N.Yuryshev, Sov. J. Quant. Electron. 14, 139 (1984). 